\title{
Die Mumienbinde des Monthemhat (Turin Cat. 1873.2) Ein weiterer Beleg für die Zusatzkapitel 173 und 174
}

\author{
Susanne Töpfer
}

The mummy bandage of Monthemhat, son of Nestanetjeretten, carries Book of the Dead Spell 133 and parts of two funerary spells which were added to the corpus of Book of the Dead spells after the Third Intermediate Period. Since Supplementary Chapters 174 and 173 - according to Pleyte's numbering - are so far attested only on papyrus Leiden $\mathrm{T} 31$, the mummy bandage must be regarded as a unique example of those compositions. The aim of the article is to make the texts on the bandage available in transliteration, translation and commentary in order to provide a basis for future Egyptological discussion about the contextualization of these supplementary chapters. It is most likely that the Pleyte spells 173 and 174 were not originally intended to be part of the Book of the Dead; rather, they must have originated as Osirian liturgies and were added into the corpus of funerary texts at a later date. The owner of the mummy bandage, named Monthemhat, seems to be identical with a member of a family of Theban priests attested from the Ptolemaic till the early Roman Period on the evidence of numerous funerary papyri and temple graffiti. According to the known genealogy of that family, Monthemhat lived in the $2^{\text {nd }}$ half of the $1^{\text {st }}$ cent. BC and/or the early $1^{\text {st }}$ cent. AD. The Turin mummy bandage Cat. 1873.2 may thus well be the latest known attestation of Book of the Dead spells on textiles.

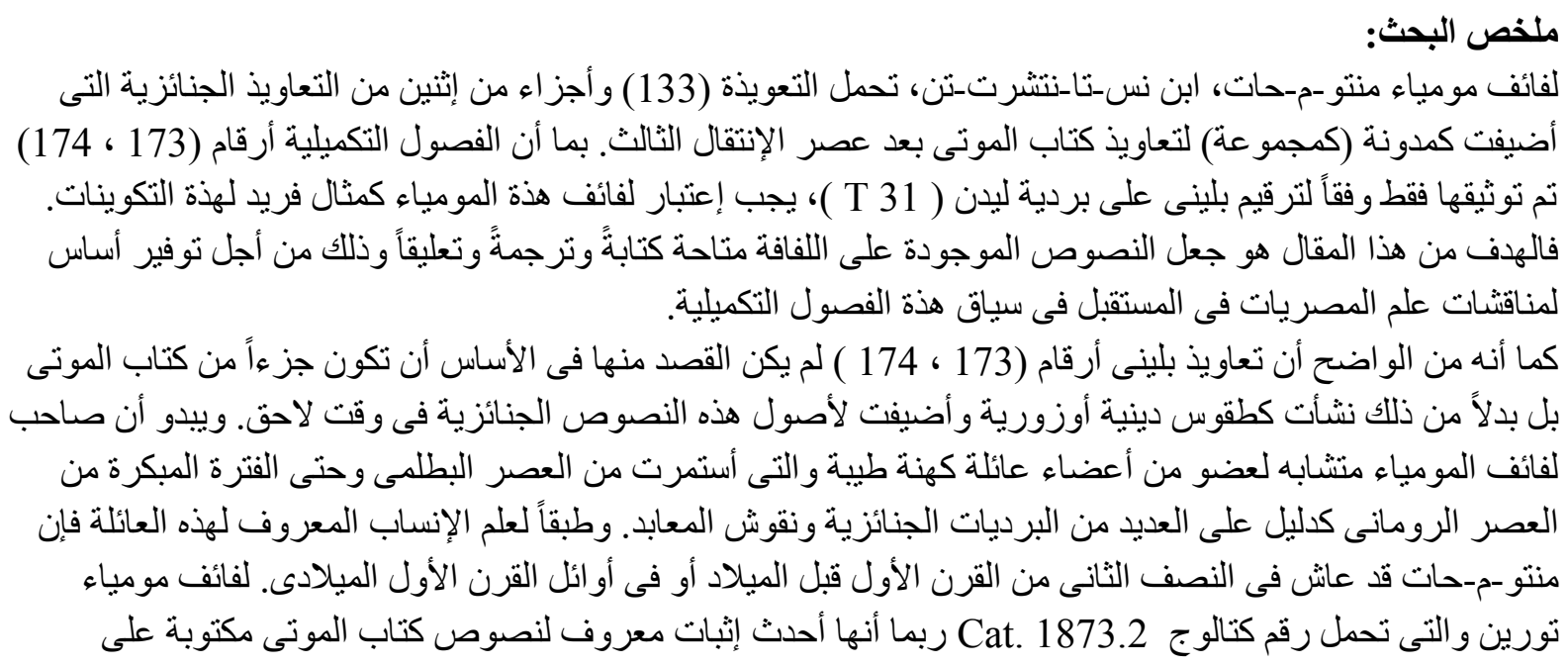

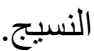

Mumienbinden mit Totenbuchsprüchen sind keine Besonderheit in der ptolemäischen Zeit und auch im Museo Egizio in Turin befinden sich mehrere Exemplare. Die Mumienbinde des Monthemhat Turin Cat. 1873.2 ist allerdings von der Masse insofern hervorzuheben, als dass es sich hierbei zum einen um das „mutmaßlich späteste Zeugnis für Totenbuch-Texte auf Mumienbinden ${ }^{* 1}$ handelt und hiermit zum anderen ein weiterer Beleg für die Zusatzkapitel 174 und 173 vorliegt. Mit Ausnahme von pLeiden T 31 sind die Zusatzkapitel 173 und 174 des Totenbuches weitestgehend unbelegt, wodurch die Mumien binde des Monthemhat einen durchaus zentralen Stellenwert für die Totenbuchforschung einnimmt resp. einnehmen wird - insbesondere, wenn man bedenkt, dass die Totenbuchsprüche auf dem Leidener Papyrus mit Ausnahme von Pleytes Edition von 1881 unpubliziert sind. $^{2}$

Ziel des Beitrages ist es, die Texte auf der Mumienbinde zugänglich zu machen, um sich in der zukünf- 
tigen Totenbuchforschung auch mit der Situierung der Zusatzkapitel 173 und 174 auseinandersetzen zu können. ${ }^{3}$ Wie gezeigt werden soll, handelt es sich hierbei mit großer Wahrscheinlichkeit um Texte, die ursprünglich aus dem Osiriskult stammen und wohl erst in ptolemäischer Zeit in das Totenbuch-Korpus integriert wurden. ${ }^{4}$

Abb. 1 Mumienbinde Cat. 1873.2 - Schriftseite. Foto Nicola Dell'Aquila/Museo Egizio.

Abb. 2 Mumienbinde Cat. 1873.2 - Rückseite. Foto Nicola Dell’Aquila/Museo Egizio

\section{Generelle Bemerkungen}

Die Mumienbinde (Abb. $1+2$ ) gelangte $1824 \mathrm{mit}$ zahlreichen anderen Objekten der Sammlung Drovetti nach Turin. Sie besteht heute aus zwei Teilen, die modern zusammengenäht worden sind; die Länge des ersten Streifens beträgt $382 \mathrm{~cm}$, die des zweiten ca. $55 \mathrm{~cm}$; die Überlappung misst ca. $1 \mathrm{~cm}$. Somit ergibt sich eine Gesamtlänge von $436,0 \mathrm{~cm}$ und eine durchschnittliche Höhe von $3,5 \mathrm{~cm}$. Anfang und Ende des ersten Bindenstreifens weisen einen glatten Schnitt auf, während der Anfang des zweiten Streifens ausgefranst ist. Das Ende des zweiten Streifens wurde zudem - ebenfalls modern - ca. 1 cm umgeklappt. Beim Zusammennähen der beiden Streifen wurde keine Rücksicht auf den Text genommen, weshalb der Name des Besitzers am Anfang des zweiten Bindenstreifens teilweise überdeckt ist. Es ist recht unwahrscheinlich, dass die beiden Abschnitte ursprünglich an dieser Stelle direkt zusammengeschlossen waren, denn der Text auf dem ersten Streifen endet abrupt mit Tb-Spruch 133 während der zweite Streifen mit Tb-Zusatzspruch 173 fortfährt. Die Textilbeschaffenheit spricht allerdings dafür, dass beide Streifen zumindest zur gleichen Binde gehören. Es gibt Belege für schmale Mumienbinden mit einer Textzeile, die bis zu $800 \mathrm{~cm}$ lang sind. ${ }^{5}$ Dementsprechend könnte zwischen den Sprüchen 133 und 173 (Pleyte) mindestens ein weiteres Kapitel gestanden haben. Es gilt allerdings zu bedenken, dass diese beiden Sprüche die Fahrt in der Sonnenbarke thematisieren und somit inhalt- lich gut zusammenpassen. Unklar bleibt, wann die Mumienbinde zerschnitten wurde resp. die beiden Abschnitte zusammengenäht worden sind. Naheliegend ist die Überlegung, dass dies entweder beim Auswickeln der (heute verlorenen) Mumie erfolgte oder Anfang des 19. Jahrhunderts auf dem Antikenmarkt, um mehrere Teile verkaufen zu können. ${ }^{6}$

Die Mumienbinde weist vereinzelt schwarze Flecken auf, bei denen es sich um Rückstände von Bitumen handelt. Zudem sind insbesondere am Anfang und am Ende des ersten Streifens sowie am Ende des zweiten Streifens braune Verfärbungen erkennbar, die recht wahrscheinlich ebenfalls auf Balsamierungsflüssigkeiten zurückzuführen sind. ${ }^{7}$

Wenngleich die Mumienbinde bisher unpubliziert gewesen ist, so war sie keineswegs unbekannt. Sie ist bereits bei Fabretti ${ }^{8}$ verzeichnet und von Quaegebeur ${ }^{9}$ sowie Coenen ${ }^{10}$ besprochen worden und in Folge dessen bei Kockelmann ${ }^{11}$ mit aufgenommen. Unter der Inventarnummer 1873 sind bei Fabretti zwei Textilstreifen verzeichnet: zunächst die Binde des Monthemhat gefolgt von der Binde eines hpt$w d \underline{d}$.t $t$-Priesters namens $P_{3}$-šrj-tz-jh.t. ${ }^{12}$ Dieser Umstand führt dazu, dass die Binde des Monthemhat in der Forschungsliteratur zumeist unter der Inventarnummer Turin 1873.1 angeführt wird, doch tatsächlich inventarisiert ist sie heute als Cat. 1873.2.

Die Binde weist auf einer Seite eine einzelne hieratische Textzeile auf. Die Paläografie der durchschnittlich $1,15 \mathrm{~cm}$ hohen Zeichen entspricht am ehesten den Charakteristiken der fortgeschrittenen ptole- 
mäischen bis frühen römischen Zeit. Aufgrund der Textilstruktur nicht immer eindeutig voneinander zu unterscheiden sind die hieratischen Schreibungen für (Y1) $\bigcirc(\mathrm{X} 1)$ und II (N23). Weiterhin zu erwähnen ist, dass der Schreiber gelegentlich (G7) zu । (Z1) verkürzt und $\odot$ (N5) stets ohne Punkt schreibt.

Bei Kockelmann sowie im Totenbucharchiv findet sich die Angabe, dass die Binde (Abb. 1) neben den Totenbuchspruch 130 einen nicht identifizierten Text außerhalb des Totenbuchs trägt. ${ }^{13}$ Die Möglichkeit, den Originaltext studieren zu können, ergibt allerdings eine andere Identifizierung der Sprüche: es handelt sich zum einen um den Totenbuchspruch 174 - Zusatzkapitel nach Pleyte - und zum anderen um den seit dem Neuen Reich belegten Totenbuchspruch 133 mit dem Ende von Zusatzkapitel 173.

\section{Transliteration und Übersetzung Spruch 174 (Pleyte) (Taf. 1)}

Der erste Abschnitt der Mumienbinde beinhaltete das Zusatzkapitel 174 des Totenbuchs. Die m.W. einzige publizierte Parallele dieses Spruches findet sich auf dem ptolemäerzeitlichen Papyrus der T3-šrj.t-t3-jh.t.t im Rijksmuseum van Oudheden zu Leiden (pLeiden T 31, Kol. IV, Z. 35-43), ${ }^{14}$ wobei die beiden Fassungen leicht voneinander abweichen. Das zentrale Motiv des Spruches ist die Regeneration der Sinnesorgane des Verstorbenen, konkret der Augen, Ohren, Nase und Zunge. Die Aktivierung der Atemfähigkeit scheint von besonderer Bedeutung zu sein, denn die Nase des Verstorbenen wird mit der des Nefertem identifiziert. Die Erwähnung des Nefertem passt gut in den Kontext der Restitution, wird dieser Gott doch mit dem Lotos verbunden, dem wohl wichtigsten Symbol für Regeneration und Wiederauferstehung im Jenseits. ${ }^{15}$ Die Aktivierung der Sinnesorgane wie auch die am Ende des Spruches erwähnte Gliedervereinigung ist ein Ziel der Balsamierung und auf eben diese Situation wird hier durch Anubis als Rezitator verwiesen. Gerade hieraus ergibt sich der Eindruck, dass der Zusatzspruch 174 aus dem Osiriskult übernommen wurde resp. aus dem Textbestand osirianischer Ritualkompositionen schöpft, welche insbesondere im späten 4. sowie 3 . Jh. v. Chr. für den Verstorbenen adaptiert worden sind, die insgesamt doch weitaus älter sind. ${ }^{16}$ Dies wird zudem daran deutlich, dass der Verstorbene sowohl im Spruchtitel als auch in der Nachschrift zu Zusatzkapitel 174 auf pLeiden T 31 als $p_{3}$ hnt.j-jmn.tjw ,der Chontamenti“ angerufen wird, wobei es sich um das klassische Epitheton des Gottes in Osirisliturgien handelt.

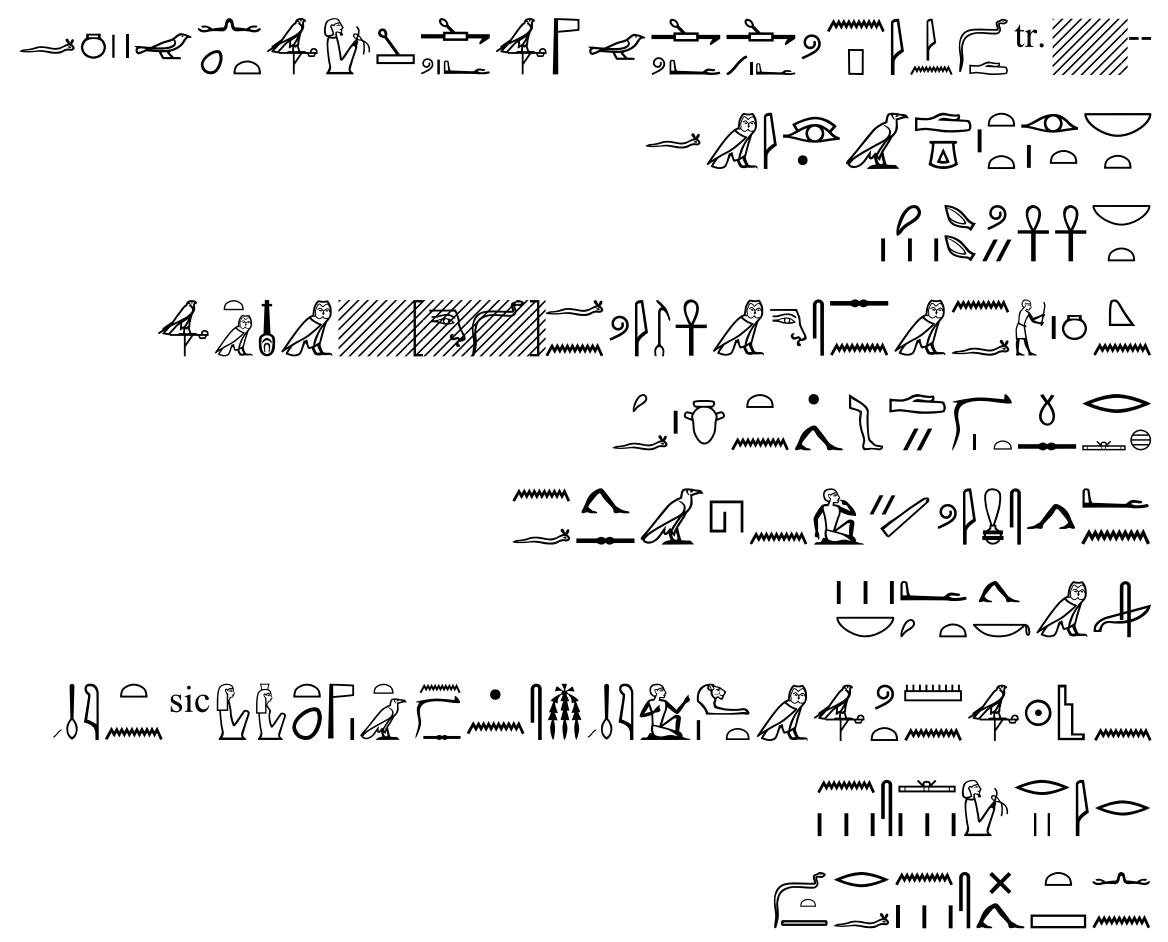

$[\ldots]^{\text {tr. a) }} d d j n J n p w^{\text {b) }}$

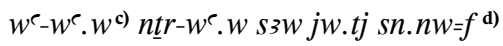

$[\ldots]^{\text {tr. }} \mathrm{Zu}$ sprechen durch Anubis:

Allereinziger, einziger Gott, Schützer ohne 


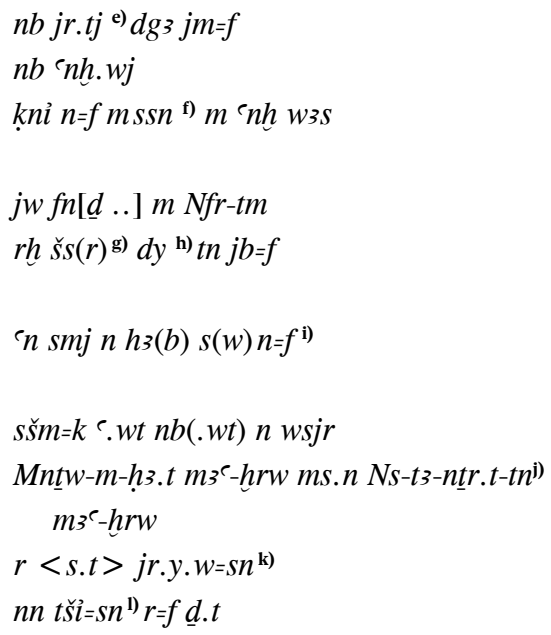

a) Am Anfang sind noch Farbspuren erkennbar, die allerdings aufgrund der braunen Verfärbung keine sichere Zeichendeutung zulassen. Gemäß der Parallele pLeiden T 31 ist das Wort sch zu erwarten, womit der Spruchtitel endet.

b) Die leicht geschwungene Form des hieratischen Zeichens am Ende des Götternamens entspricht im Grunde vielmehr $w$ statt $(\mathrm{G} 7)$.

c) Die Form des zweiten Harpunenzeichens (T21) sieht vielmehr wie $\Longleftarrow(\mathrm{O} 29)$ aus, doch der mit dem $w$ verbundene Hacken am Ende macht die eigentliche Bedeutung klar. Ebenso ist das drit-

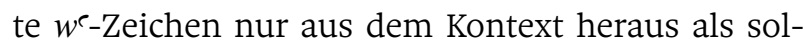
ches zu verstehen, denn der Hacken und der Ideogrammstrich bilden ein Zeichen.

d) Die Abfolge der Epitheta des Osiris-Chontamenti stimmt mit der in pLeiden $\mathrm{T} 31$ überein, dort fehlt allerdings die Gruppe nach $n \underline{t} r-w^{e} . w$, die sehr wahrscheinlich $s 3 w$ „Schützer, Wächter“ (Wb III, 418.1-4) $\mathrm{zu}$ lesen ist. Zur dieser Bezeichnung des Osiris vgl. LGG VI, 125c-126a und ferner Töpfer, Das Balsamierungsritual, 81 (Kom. $\mathrm{x}$ ).

e) Konkret die beiden Udjat-Auge sind gemeint. Die Parallele pLeiden $\mathrm{T} 31$ schreibt phonetisch $w \underline{d}$ 3.tj.

f) Die eher ungewöhnliche Schreibung Verbs snsn „atmen“ ist hervorzuheben, da die Gra-

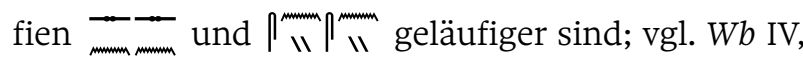
172.2-10; 277.9-16.

Zudem zu betonen ist die hieratische Form von $\sim_{\infty}=$ (O34), da diese vielmehr der Buchrolle gleicht.

Der Text der Mumienbinde weicht an dieser Stelle von pLeiden T $31 \mathrm{ab}$, dort: ‘nh.wj knis $\underline{d}$ m snỉ=k „die beiden Ohren sind tüchtig/tapfer beim Hören, mö- seinesgleichen;

Herr der beiden Augen, die mit ihm sehen;

Herr der beiden Ohren;

dem Kraft gehört beim Atmen in Leben und

Wohlergehen,

indem [seine] Nase die des Nefertem ist;

der kennt den Gedanken und jene Obliegenheit seines Herzens;

der die Anklage umwendet, gegen den, der sie aussendet gegen ihn.

Mögest du leiten alle Glieder des Osiris des

Monthemhat, gerechtfertigt, geboren von

Nestanetjeretten, gerechtfertigt,

zu ihrem entsprechenden Platz.

Nicht werden sie sich abwenden von ihm ewiglich!

gest du riechen ..... Es mag sein, dass $m$ hier eine defektive Schreibung für $s d m$ ist; in diesem Falle müssten statt einer $s \underline{d m} . n=f$-Form eine Genitiv-Konstruktion sowie eine Relativform angesetzt werden: $n b$ ¿nh.wj kni $n=f<s \underline{d}>m$ sns(n) $m \ldots$.. „Herr der beiden Ohren, die tüchtig/tapfer sind für ihn beim Hören, der atmet in.....

g) $\mathrm{Zu}$ šsr in der Bedeutung „Ausspruch, Gedanke“ insbesondere des Herzens siehe Wb IV, 548.8-13.

h) In der Übersetzung von $d y$ mit „Obliegenheit“ folge ich einem der Reviewer, der diese durchaus sinnvolle Lesung vorschlägt im Hinblick auf $r d$ (Wb II, 463.13), demot. $r t$ (CDD R, 80-81) „Position, Stellung“. Die Parallele pLeiden T 31 schreibt unklares $4 \Omega \hat{a}$, wohl beeinflusst von $j 3 \underline{t}$, schmerzen, verletzt sein“ (Wb I, 34.21-22). Dort steht zudem $m$ $j b=f$.

i) Die Parallele pLeiden $\mathrm{T} 31$ hat nur $h_{3} b=f$. Das Epitheton steht wohl im Zusammenhang mit der mythologischen Episode um die Rechtfertigung des Osiris gegen seine Feinde, insbesondere Seth.

j) Der Name der Mutter ist an dieser Stelle leicht verschrieben; das Personendeterminativ steht vor $t$.

k) Die Parallele hat $r s z w=s n$. Dementsprechend ist man geneigt zunächst $r$ jry $s 3 w=s n$,die zur ihrer Bewachung Gehörigen“ o.ä. zu lesen. Womöglich liegt hier aber auch eine unvollständige Schreibung für $r<s . t>$ jr.y.w=sn ,an ihrem entsprechenden Platz“ vor, was zumindest inhaltlich sinnvoll wäre, denn gemeint ist sicherlich die Gliedervereinigung. Konkret geht es wohl um die richtige Positionierung der oben genannten Bestandteile des Kopfes (Augen, Ohren, Nase) und damit um die Versammlung aller 
Glieder des Osiris resp. des mit diesem assoziierten Verstorbenen. $\mathrm{Zu}$ den Osirisgliedern siehe Z. 45-46 und 54-59 des Choiaktextes von Dendara sowie pJumilhac Kol. III.19-IV.28 und ferner pRhind 1, Kol. III.h3-5; hierzu mit Referenzen Töpfer, Balsamierungsritual, 235.

1) Die Parallele pLeiden T 31 hat hrj „sich entfernen“ statt $t \check{s} \grave{i}$, doch ansonsten sind die Schlussverse gleich.

\section{Spruch 133 (Taf. 2)}

Der zweite Textabschnitt ist eine verkürzte Fassung von Totenbuchspruch 133, in dessen Mittelpunkt die Teilnahme des Verstorbenen am täglichen Sonnenlauf steht, was zur Gewährleistung der ewigen Fortexistenz unabdingbar gewesen ist. Im zweiten
Textabschnitt spielt abermals das Motiv der Gliedervereinigung und Erneuerung eine zentrale Rolle, diesmal konkret des Re, mit welchem der Verstorbene im Spruch identifiziert wird (pTurin Cat. 1791): wsjr $N N$ wdz $m j R^{e}$ „Osiris des NN ist heil wie Re“

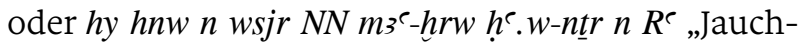
zen und Jubel dem Osiris des NN, dem Gottesleib des Re“.

Im Hinblick auf die Epitheta „Herr der beiden Augen“ und „Herr der beiden Ohren“ im zuvor genannten Zusatzkapitel 174 ist hervorzuheben, dass gerade die Restitution der Augen und Ohren in Tb 133 des Verstorbenen gepriesen wird (pTurin Cat. 1791): nfr.wj m33 $m$ jr.tj sdm $m$ ms $\underline{d}$ r.wj „wie schön ist es, mit den Augen zu sehen und mit den Ohren zu hören“.

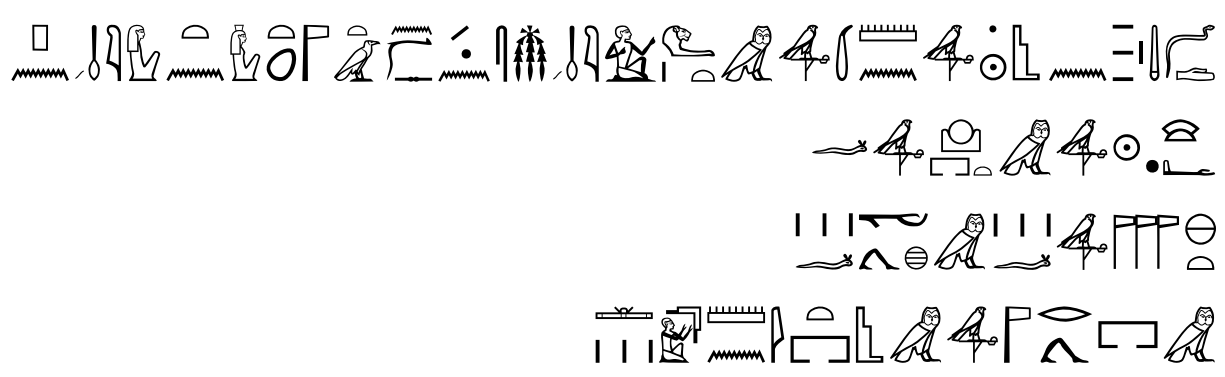

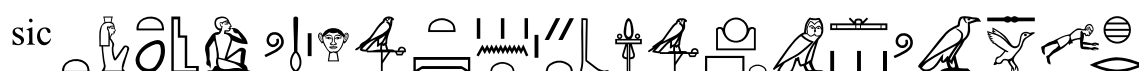

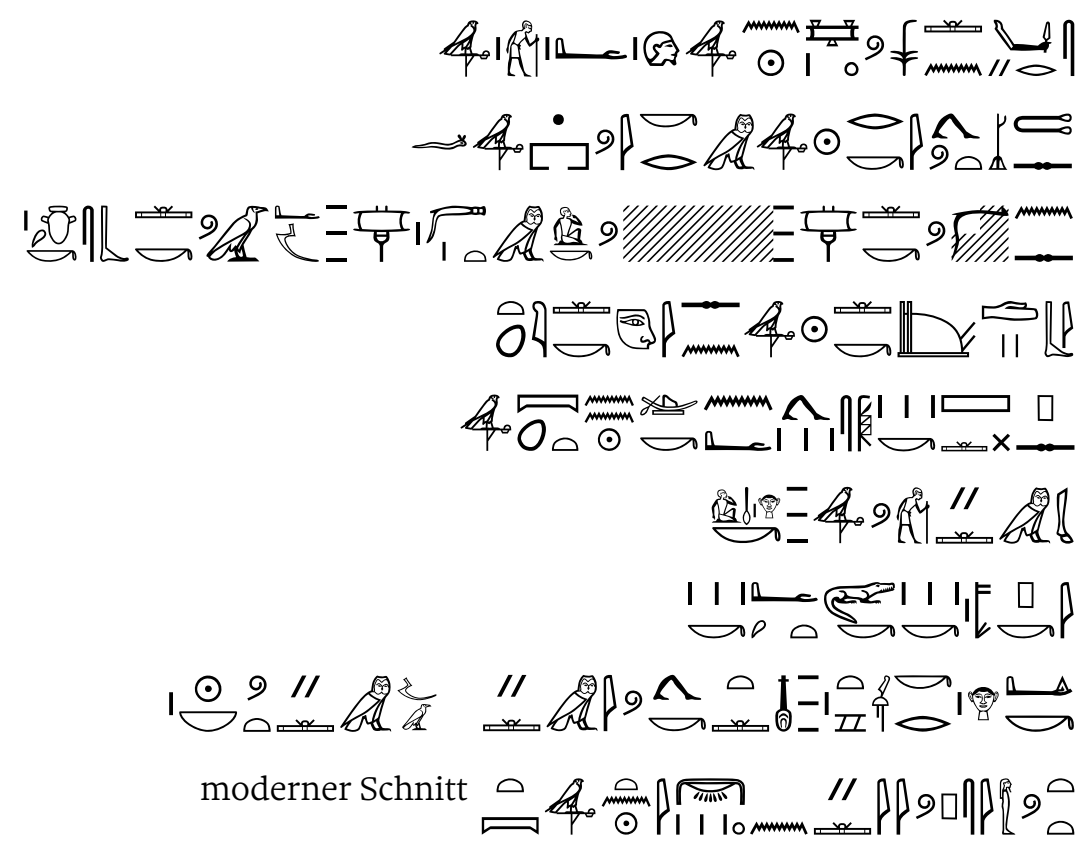

$\underline{d} d-m d w n w s j r$

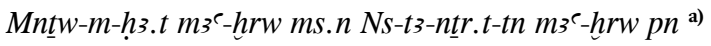

hei $R^{e} m$ 3h.t=f

$p s \underline{d} . t=f m-\underline{h} . t=f$
Rezitation durch Osiris des

Monthemhat, gerechtfertigt, geboren

von Nestanetjeretten, gerechtfertigt, diesen:

Es erscheint Re in seinem Horizont,

seine Neunheit ist hinter ihm, 


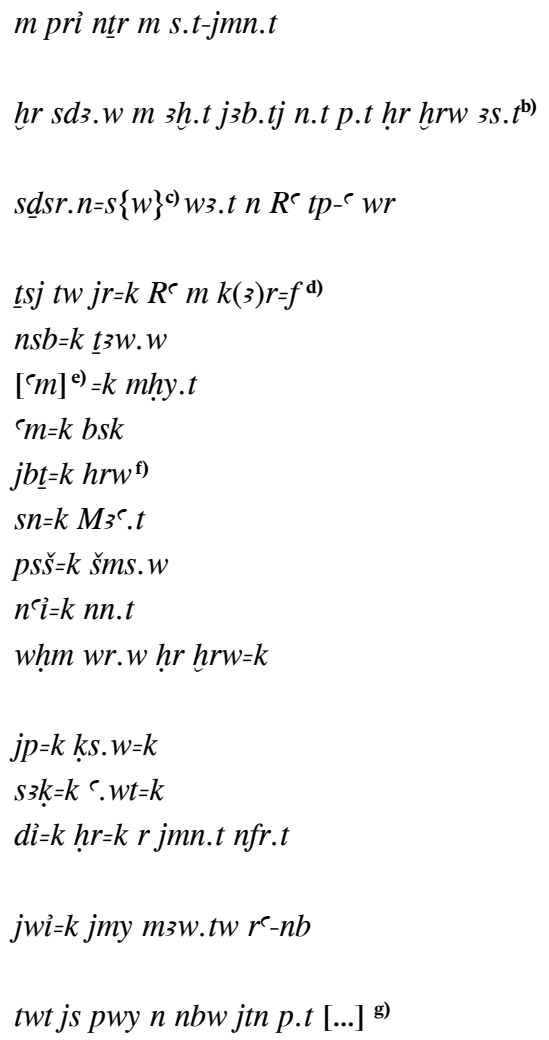

a) Das Demonstrativpronomen bezieht sich auf den Verstorbenen.

b) Es steht tatsächlich ein weiteres - redundantes - $t$ nach dem Göttinnendeterminativ. Die Versionen des Neuen Reiches haben statt „Isis“ vielmehr „Nut“, die Personifikation des Himmels. Betrachtet man die ursprüngliche Bedeutung des Spruches dann wird deutlich, dass der Sonnenaufgang im Osten mit der Geburt des Re durch Nut verglichen wird, die - einer Gebärenden entsprechend - dabei wohl Klagegeschrei ausstößt, was hier mit der „Stimme“ gemeint sein dürfte. In den späten Texten liegt der Schwerpunkt offensichtlich auf dem Aspekt der Klage, denn im Osiriskult gilt Isis als Klagefrau par excellence. Siehe hierzu auch die Ausführungen von Pries, Stundenwachen, 116-117 zu $r / h r$ hrww=f „auf meine Stimme hin“ und dem semantisch ähnlichen $r / h r j w j=f$ „auf meine Klage hin“.

c) Die Parallelen haben $s \underline{d} s r . n=s$ „sie hat prächtig gemacht/ausgestattet“. Dementsprechend sollte $s w$ hier als späte Schreibung für $s$ angesehen werden. d) Das Suffixpronomen $=f$ ist nur sehr schwach $\mathrm{zu}$ erkennen, da der Schreiber erst nach dem Vers neu in die Farbe eintaucht. Die Parallelen schreiben jmj „befindlich in (seinem Schrein)“. Gemeint ist der Sonnengott in seinem Pavillon auf der Barke. wenn der Gott herauskommt aus dem geheimen Platz.

Es fällt ein Zittern nieder im östlichen Horizont des Himmels auf die Stimme der Isis hin.

Sie hat den Weg prächtig sein lassen für Re, den großen Vorfahren.

Erhebe dich doch, Re, in seinem Schrein!

Mögest du verschlingen die Winde.

Mögest du verschlucken den Nordwind.

Mögest du verschlucken das Herz.

Mögest du einfangen den Tag.

Mögest du küssen die Maat.

Mögest du teilen das Gefolge.

Mögest du befahren den Gegenhimmel

Mögen die Großen (das Gesagte) wiederholen auf deine Stimme hin.

Mögest du deine Knochen zählen.

Mögest du deine Glieder zusammenfügen.

Mögest du dein Gesicht dem schönen Westen zuwenden.

Mögest du dorthin kommen, indem du täglich erneuerst wirst.

Du bist schließlich dieser aus Gold, die Scheibe des Himmels [...]

e) Der Text ist hier aufgrund der Bitumenreste unleserlich. Ergänzung nach den Parallelen.

f) Da Ra selbst hier angesprochen wird, ist die Gruppe vielmehr $h r w$ „Tag“ statt $r^{c}$ „Sonne“ zu lesen.

g) Der gesamte Vers ist verkürzt wiedergegeben, es müsste heißen (nach pTurin Cat. 1791): twt js twt pw $n$ nbw hr sm3.w n jtn $m$ p.t $\underline{h} r$ sd3 „Du bist schließlich dieses Abbild aus Gold unter den Zweigen der Scheibe im Himmel unter Zittern.“ Die hieratische Schreibung des Personalpronomens twt ( $W b \mathrm{~V}$, 360.5-9) entspricht der Grafie für $t w t$ „Abbild“ (Wb V, 255.8-256.20), was nicht ungewöhnlich ist für späte Schreibungen. Unklar ist, wie der Text weitergeht, denn die Binde ist an dieser Stelle zu Ende und der nächste Streifen, der modern angefügt wurde, setzt mit dem Namen des Nutznießers ein.

\section{Spruch 173 (Pleyte) (Taf. 3)}

Das Thema des kurzen Zusatzkapitels 173 ist ebenfalls die Gliedervereinigung und somit die Vervollständigung des Körpers. Der Hintergrund des Spruches ist die Erscheinung des regenerierten Gottes resp. Verstorbenen mit der Sonne bei Tagesanbruch, worauf anhand des Spruchtitels geschlossen werden kann (pLeiden T 31, Kol. IV, Z. 29-30): r3 $n$ dì.t prỉ p3 šn.w n re tp n nз ms.w Hr $r$ dwz.t „Spruch um zu ver- 
anlassen, dass der Umkreis der Sonne herauskommt an der Spitze der Horuskinder zur Grabkammer.“ Es sind die Horuskinder, welche die Mumie am Ende der Nachtwache am Morgen mit den ersten Sonnenstrahlen von der Balsamierungshalle zum Grab tragen. ${ }^{17}$ Der Kontext des Spruches ist demzufolge die Stundenwachen,${ }^{18}$ konkret die erste Tagesstunde dürfte gemeint sein, denn im Text heißt es (pLeiden T 31, Kol. IV, Z. 30-32): j Hr nb ph.ww jni.n=k nn gmi. $n=k n n w n w . t=k$ wn $j t=k h t m m(j) h . t=f$,Oh Horus, Herr der Enden (des Landes), du hast diese gebracht, du hast diese gefunden zu deiner Stunde des Öffnens. Dein Vater ist vervollständigt mit seinen Reliqui- en." Es bestehen zweifellos Anklänge an die osirianischen Stundenwachenriten, in denen Horus zum Schutz seines Vaters agiert, weshalb auch Zusatzkapitel 173 aus dem Osiriskult übernommen worden sein dürfte. ${ }^{19}$ Den Text auf Tb 133 folgen zu lassen ist schließlich plausibel, da beide Sprüche die körperliche Restitution sowie die Erscheinung resp.Vereinigung mit dem Sonnengott in seinem täglichen (Re) und nächtlichen (Atum) Aspekt thematisieren. ${ }^{20}$ Somit kann die Vermutung aufgestellt werden, dass auf dem verlorenen Teil des Textilstreifens der Anfang von Zusatzkapitel 173 und vielleicht noch ein Abschnitt von $\mathrm{Tb} 133$ gestanden haben könnte.

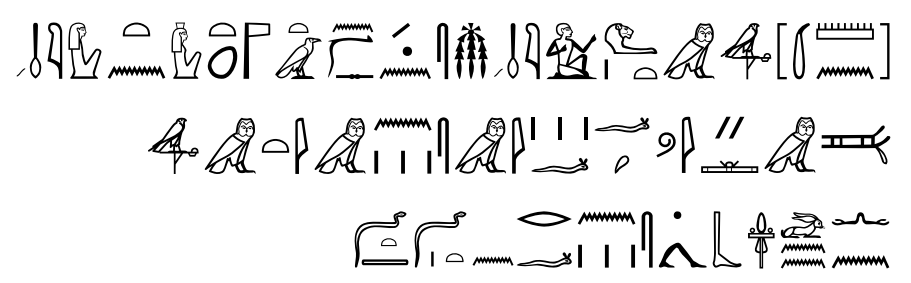

$[M n t w]^{\mathrm{a})}-m-\underline{h} 3 . t m 3^{\mathrm{C}}-h \mathrm{hr} w \mathrm{~ms} . n$

Ns-t3-ntr.t-tn mie-hrrw

$t m j w f=f j m=s n^{\text {b) }} m j t m^{\text {c) }}$

$n n-w n n j z b=s n \quad r=f n-\underline{d} . t-\underline{d} . t$
[Month]emhat, gerechtfertigt, geboren von Nestanetjeretten, gerechtfertigt. Vollständig ist sein Fleisch durch sie wie Atum. Nicht sollen sie zurückweichen von ihm ewiglich! a) Hier schließt der zweite Textilstreifen mit dem Ende von Spruch 173 (Pleyte) an, der mit dem Namen des Nutznießers beginnt. Der Streifen wurde modern ohne Rücksichtnahme auf den Text zusammengenäht und vom ersten Namensbestandteil Mntw ist lediglich das Götterdeterminativ noch erhalten, das allerdings vom ersten Streifen überdeckt ist.

b) Mit =sn sind die Gaben gemeint, die Horus zu Beginn von Tb 173 (Pleyte) zum Schutz des Verstorbenen heranbringt. Gemäß pLeiden T 31 handelt es sich hierbei um jh.t, eine Bezeichnung der „Opfergaben“ aber auch der „Reliquien“. Da sowohl in Tb 174 (Pleyte) wie auch in Tb 133 die Wiederherstellung der Sinnesorgane und die Gliedervereinigung zentrale Themen sind, dürfte sich das Pronomen auf die Körperteile beziehen.

c) Bereits in den Pyramidentexten werden der Leib bzw. einzelne Glieder - mit Ausnahme des Gesichts - des Verstorbenen (Königs) mit Atum identifiziert; vgl. PT 213 (§134a-135c), PT 537 (§1298b), PT 690 (§2098a). Eine Passage in PT 215 enthält eine „Gliedervergottung“ (\$148a-149c), in welcher die Augen und Ohren des Verstorbenen mit den beiden Kindern des Atum (Schu und Tefnut) gleichgesetzt werden, was insofern von Interesse ist, als dass gerade diese beiden Sinnesorgane in $\mathrm{Tb} 174$ (Pleyte) und $\mathrm{Tb}$ 133 eine wichtige Rolle einnehmen.

\section{Besitzer}

Der Besitzer der Mumienbinde trägt den Namen Mntw-m-ḩz.t (PN I, 154.7; DemNB, 597) und er wird insgesamt dreimal genannt, stets in Verbindung mit seiner Mutter 든 Ns-t3-ntr.t-tn (PN I, 179.19). Mit Ausnahme der Verstorbenenbezeichnung wsjr sind keine Titel wiedergegeben. In der Forschung wird der Besitzer der Mumienbinde mit dem Besitzer des Funerärpapyrus Tübingen $2016^{21}$ namens Monthemhat gleichgesetzt, da der Name seiner Mutter ebenfalls Nesta- 
netjeretten lautet. Die Gleichsetzung erfolgt allein aufgrund des Mutternamens, denn die Belege sind in der griechisch-römischen Zeit überschaubar; ${ }^{22}$ lediglich auf der Mumienbinde Turin Cat. 1873.2 und pTübingen 2012 kommt diese Filiation vor. Als ein weiteres Indiz für eine mögliche Identifikation der Besitzer ist der Charakter der Texte auf dem Textil sowie dem Papyrus anzuführen. Papyrus Tübingen 2012 beinhaltet eine singuläre Textkomposition bestehend aus Totenbuchsprüchen, einem Götterdekret sowie einer Kurzfassung vom „Buch vom Atmen“. Das Dekret (Kol. XIII.7-8) richtet sich an Osiris-Wennefer, Verfasser des Textes war ursprünglich Amun-Re. Dieser wird nicht namentlich genannt, doch folgen nach dem Namen des Osiris-Wennefer die typischen Epitheta des Amun, die hier aber mit den Osiris-Bezeichnungen verschmelzen. Das Dekret leitet Sprüche aus dem Umkreis der „Bücher vom Atmen“ ein, die mit dem Wunsch nach Teilnahme am Dekadenfest beginnen (Kol. XIII.910), wodurch die Verortung nach Theben deutlich wird. Monthemhat hat sich mit pTübingen 2012 demzufolge eine durchaus innovative Textkomposition mit ins Grab genommen. Und auch für die Beschriftung der Mumienbinde wird der Kanon an Totenbuchsprüchen durchbrochen, indem mit den Zusatzkapiteln 173 und 174 Texte aus dem Osiriskult auswählt wurden, die in adaptierter Form in das Totenbuch-Korpus integriert werden. Die Tatsache, dass zwei Funerärobjekte belegt sind, die einem

\section{Endnoten}

${ }^{1}$ Zitat Kockelmann, Untersuchungen, 20.

${ }^{2}$ Pleyte, Chapitres supplémentaires.

${ }^{3}$ Für kritische Hinweise und Anregungen herzlich $\mathrm{zu}$ danken ist zum einen den beiden Reviewern und zum anderen Federico Poole; ihm als Herausgeber zudem für die Annahme des Beitrages.

${ }^{4}$ Wie es zunehmend mit den Zusatzkapiteln 162 bis 167 erfolgt, hierzu Wüthrich, Eléments de théologie thébaine; Dies., Édition synoptique; Dahms, Pehal und Willems, JEA 100, 395-420 und Quack, SAK 45, 283-293.

${ }^{5}$ Vgl. die Belege bei Kockelmann, Untersuchungen, 87-88.

${ }^{6}$ Ein durchaus häufiger Vorgang, siehe hierzu die Ausführungen von Kockelmann, Untersuchungen, 5-7 zu Mumienbinden als Sammelobjekt.

${ }^{7} \mathrm{Zu}$ hieraus resultierende Rückschlüsse auf die Anbringungsweise von Mumienbinden siehe
Mann namens Monthemhat geboren von einer Frau namens Nestanetjeretten zugeordnet werden können, und die sich beide im Textbestand insofern von geläufigen Totenbuchhandschriften unterscheiden, als dass sie „neue“ Texte integrieren, spricht m.E. für die Hypothese, in dem Besitzer beider Objekte die gleiche Person zu sehen.

Für die zeitliche Verankerung beider Textabschriften ist schließlich noch zu bemerken, dass pTübingen 2012 mit einer Genealogie endet, in welcher Monthemhat sechs Generationen von Priestern des Min sowie des Amun aufzählt. Die Familienmitglieder sind durch eine Gruppe von Totenbüchern und „Bücher vom Atmen“ sowie einigen demotischen Graffiti über nunmehr insgesamt acht Generationen hinweg belegt. Auf der Mehrheit der Funerärpapyri befinden sich genealogische Angaben mit Amtsbezeichnungen der Vorfahren, auf deren Basis Coenen einen Stammbaum erstellte. ${ }^{23} \mathrm{Zu}$ den von Coenen früher datierten Manuskripten zählt insbesondere pCologny CIV (pBodmer 104), den er um 200 v. Chr. ansetzt, zu den spätesten pTübingen 2012, den er in die 2. Hälfte des 1. Jahrhunderts v. Chr. datiert. In paläografischer Hinsicht ist eine Datierung der Textabschrift auf pTübingen 2012 in das 1. Jh. v.-1. Jh.

n. Chr. möglich und damit dürfte auch die Mumienbinde Cat. 1873.2 in diesem zeitlichen Rahmen verankert werden, was sie tatsächlich zu dem wohl „spätesten Zeugnis für Totenbuch-Texte auf Mumienbinden“ ${ }^{24}$ machen würde.

Kockelmann, Untersuchungen, 225-230.

${ }^{8}$ Fabretti, Catalogo generale, 238.

${ }^{9}$ Quaegebeur, in: Bierbrier (Hg.), Portraits and Masks, 74 mit Anm. 55.

${ }^{10}$ Coenen, in: Clarysse, Schoors und Willems (Hgg.), Egyptian Religion, 1110-1111; Ders., JEA 86, 93; Ders., RdE 52, 74-79; Ders., ZÄS 130, 163.

${ }^{11}$ Kockelmann, Untersuchungen, 20, 222, $261 \mathrm{mit}$ Anm. 178 [Kat.-Nr. 111], und http://totenbuch.awk. nrw.de/objekt/tm114074.

${ }^{12}$ Fabretti, Catalogo generale, 238. Die Mumienbinde des P3-šrj-t3-jh.t (PN I 119.9-10; DemNB 262) ist heute inventarisiert als Cat. 1873.1.

${ }^{13}$ Kockelmann, Untersuchungen, 20 mit Anm. 78.

${ }^{14}$ Pleyte, Chapitres supplémentaires, 198-202, pl. 177 178. Ferner B. Backes, in: Thesaurus Linguae Aegyptiae (Stand der Datenbank: 31.10.2014). Die Umschrift und Übersetzung einzelner Passagen erfolgt nach Fotos im Totenbucharchiv Bonn: TM 56996, http://toten- 
buch.awk.nrw.de/objekt/tm56996.

${ }^{15}$ Siehe hierzu Totenbuchspruch 81 resp. 81B: $r 3$ $n$ jrì.t hpr.w $m$ sšn „Spruch, um Gestalt anzunehmen als Lotosblume“. Vom Lotos, dem Abbild (twt) des Nefertem, wird gesagt, dass er sich an der Nase des Re befindet. Vgl. auch den folgenden Totenbuchspruch 133.

${ }^{16} \mathrm{Zu}$ Osirisliturgien und ihre private Verwendung siehe die Beiträge in Backes und Dieleman (Hgg.), Liturgical texts, bes. 1-13. Zur Integration „neuer“ Texte in das Totenbuch-Korpus siehe die Edition von Backes, Drei Totenpapyri.

${ }^{17}$ Hierzu mit Verweisen Pries, Stundenwachen, 100 mit Anm. 290.

${ }^{18}$ Zur Rolle der Horuskinder in den Stundenwachen siehe Pries, Stundenwachen, 43 - 44, 101-102, 428-430.

${ }^{19}$ Zur ersten Tagesstunde siehe mit Kommentaren Pries, Stundenwachen, 339-359. Siehe ferner die Ausführungen in Töpfer, Das Balsamierungsritual, 310-316.

${ }^{20}$ Zum Schutz des Leibes durch Re-Atum siehe ferner Pries, Stundenwachen, 134.

${ }^{21}$ Edition Töpfer und Müller-Roth, Das Ende der Totenbuchtradition. Siehe hierzu ferner die (stellenweise zu Recht) kritische Rezension von Mosher, BiOr 5-6, 655-664.

${ }^{22} \mathrm{Zu}$ den Belegen siehe Töpfer und Müller-Roth, Das Ende der Totenbuchtradition, 102 mit Anm. 385.

${ }^{23}$ Zur Genealogie mit Verweisen auf die grundlegenden Studien von Marc Coenen siehe Töpfer und Müller-Roth, Das Ende der Totenbuchtradition, 9-11, 103-105, 109 und Töpfer, Das Balsamierungsritual, 51-57.

${ }^{24}$ Kockelmann, Untersuchungen, 20. Wenngleich diese Aussage zu relativieren ist, da die Zusatzkapitel 173 und 174 jüngere Adaptionen älterer Texte sind, die dem Totenbuch schließlich erst später - wohl in ptolemäischer Zeit - zugesetzt wurden.

\section{Bibliografie}

Backes, B. und J. Dieleman, (Hgg.), Liturgical Texts for Osiris and the Deceased in Late Period and Greco-Roman Egypt (Studien zur spätägyptischen Religion 14), Wiesbaden 2015.

Backes, B., Drei Totenpapyri aus einer thebanischen Werkstatt der Spätzeit (pBerlin P. 3158, pBerlin P. 3159, pAberdeen ABDUA 84023) (Handschriften des Altägyptischen Totenbuches 11), Wiesbaden 2009.

Coenen, M., "The Funerary Papyri of Horos Son of Estneteretten in the Kunsthistorisches Museum in Vienna”, ZÄS 130 (2003), 160-169.

Coenen, M., "On the Demise of the Book of the Dead in Ptolemaic Thebes”, RdE 52 (2001), 69-84.

Coenen, M., "The Funerary Papyri of the Bodleian Library at Oxford", JEA 86 (2000), 81-98.

Coenen, M., "The Dating of the Papyri Joseph Smith I, $\mathrm{X}$ and XI and Min Who Massacres His Enemies", in: W. Clarysse, A. Schoors und H. Willems (Hgg.), Egyptian Religion. The last thousand Years. Studies dedicated to the Memory of Jan Quaegebeur (OLA 84-85), Leuven 1998, 1107-1115.

Dahms, J., M. Pehal und H.Willems, "Ramses II helps the Dead: An interpretation of Book of the Dead supplementary chapter 166", JEA 100 (2014), 395-420.

Fabretti, A., F. Rossi und R.V. Lanzone, regio Museo di Torino. Antichità Egizie, Band 1 Catalogo generale dei musei di antichità e degli oggetti d'arte raccolti nelle gallerie e biblioteche del regno, 1. Piemonte, Torino 1882.

Kockelmann, H., Untersuchungen zu den späten Totenbuch-Handschriften auf Mumienbinden Bd. II (Studien zum Altägyptischen Totenbuch 12), Wiesbaden 2008.

Lüddeckens, E., Demotisches Namenbuch, Wiesbaden 1980.

Mosher, M., "Rezension zu Töpfer und Müller-Roth, Das Ende der Totenbuchtradition”, BiOr 5-6 (2014), 655-664.

Pleyte, W., Chapitres supplémentaires du Livre des Morts 162 à 174. Traduction et Commentaire, Leiden 1881.

Pries, A.H., Die Stundenwachen im Osiriskult. Eine Studie zur Tradition und späten Rezeption von Ritualen im Alten Ägypten (Studien zur spätägyptischen Religion 2), Wiesbaden 2011.

Quack, J.F., “Zur Situierung von TB 166 Pleyte”, SAK 45 (2016), 283-293.

Quaegebeur, J., "Books of Thoth Belonging to Owners of Portraits? On Dating Late Hieratic Funerary Papyri”, in: M.L. Bierbrier (Hg.), Portraits and Masks. Burial Customs in Roman Egypt, London 1997, 72-77.

Ranke, H., Die ägyptischen Personennamen, Bd. I., Glückstadt 1935.

Töpfer, S., Das Balsamierungsritual. Eine (Neu-)Edition der Textkomposition Balsamierungsritual (pBoulaq 3, pLouvre 5158, pDurham 1983.11+pSt. Petersburg 18128) (Studien zur spätägyptischen Religion 13), Wiesbaden 2015.

Töpfer, S. und M. Müller-Roth, Das Ende der Totenbuchtradition und der Übergang zum Buch vom Atmen. Die Totenbücher des Monthemhat (pTübingen 2012) und der Tanedjmet (pLouvre N 3085) (Handschriften des Altägyptischen Totenbuchs) 13, Wiesbaden 2011.

Wüthrich, A., Édition synoptique et traduction des chapitres supplémentaires du Livre des Morts 162 à 167 (Studien zu Altägyptischen Totentexten 19), Wiesbaden 2015.

Wüthrich, A., Eléments de théologie thébaine: les chapitres supplémentaires du Livre des Morts (Studien zum Altägyptischen Totenbuch 16), Wiesbaden 2010. 


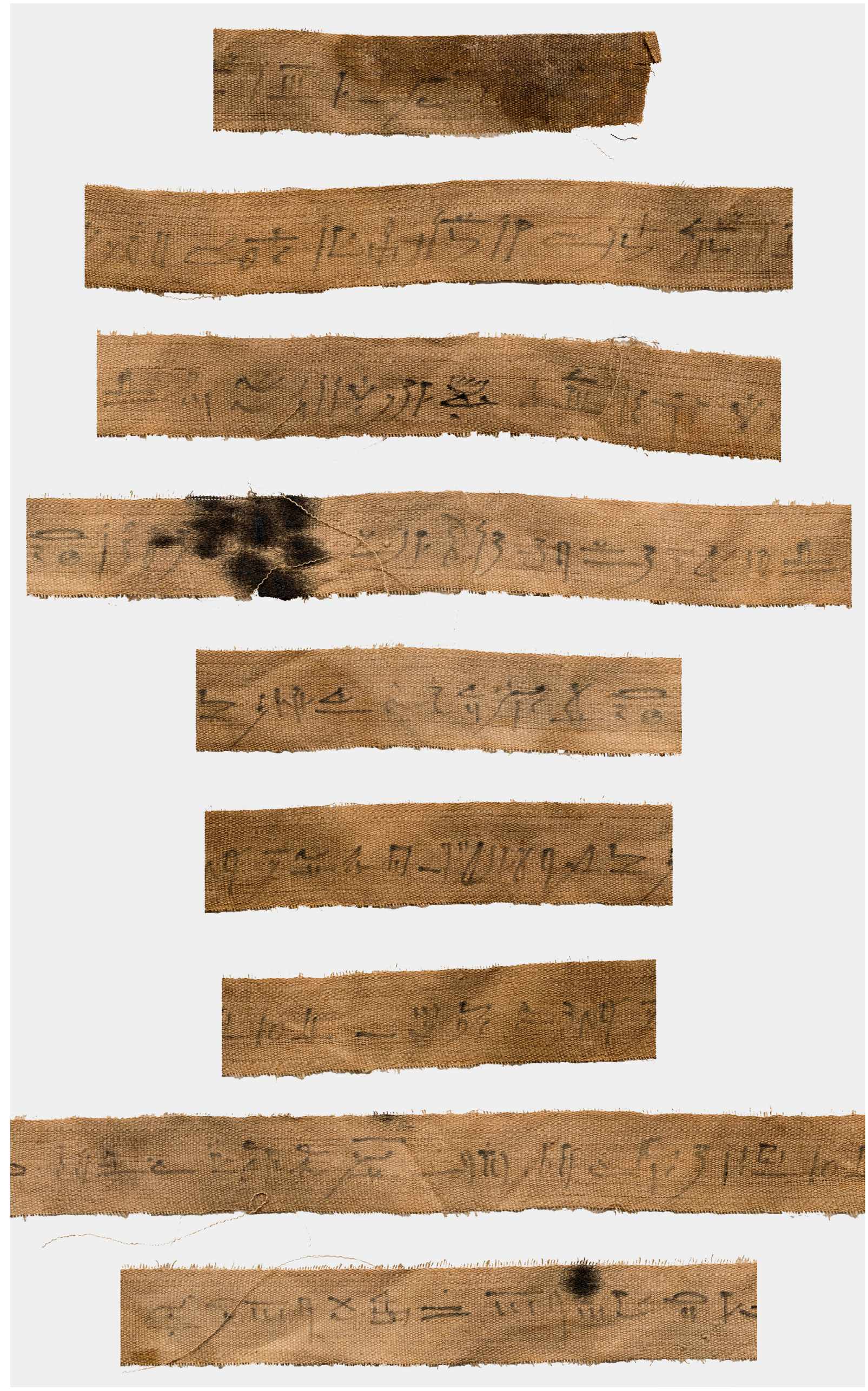

Taf. 1 Spruch 174 (Pleyte) 


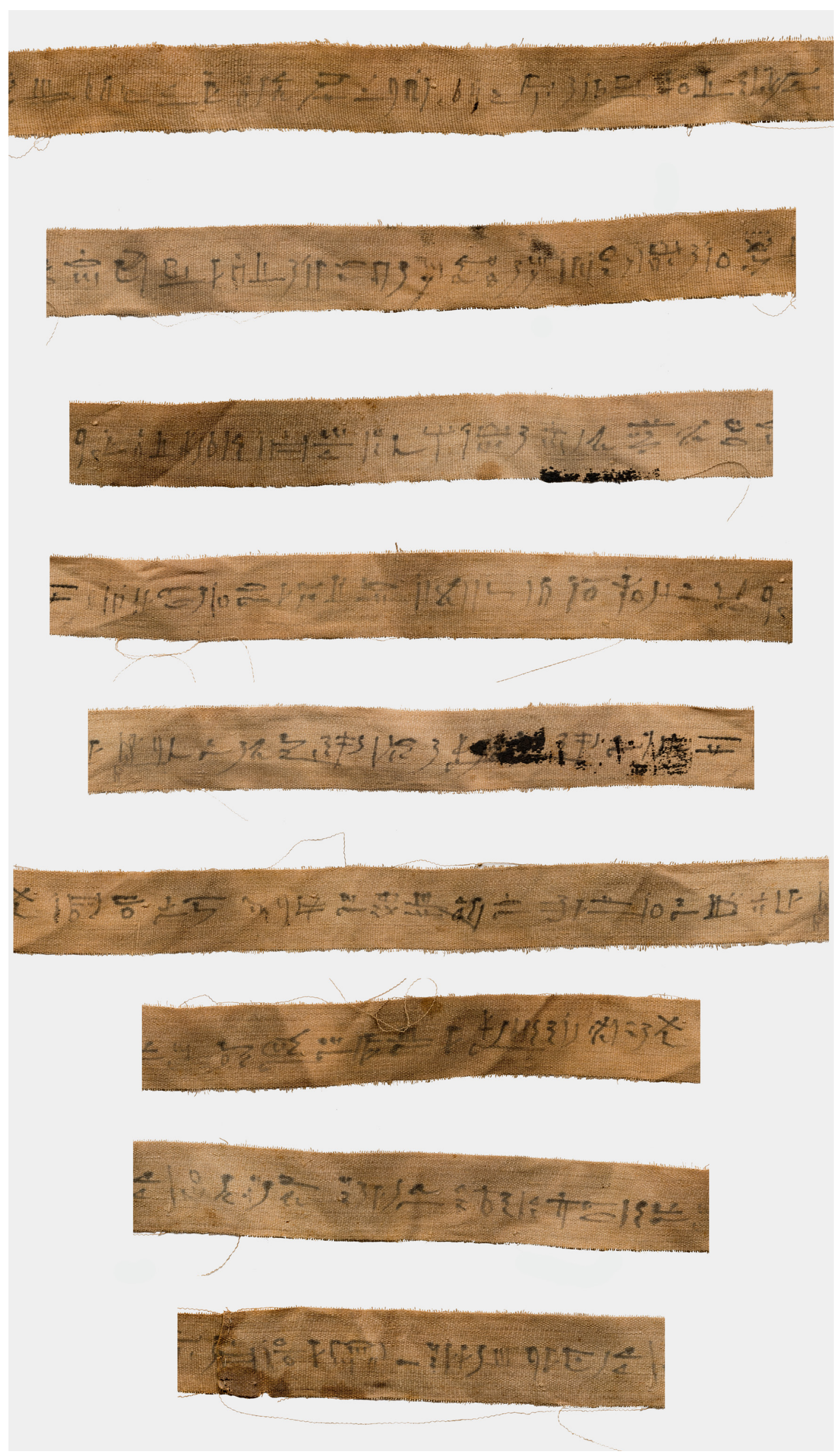

Taf. 2 Spruch 133 


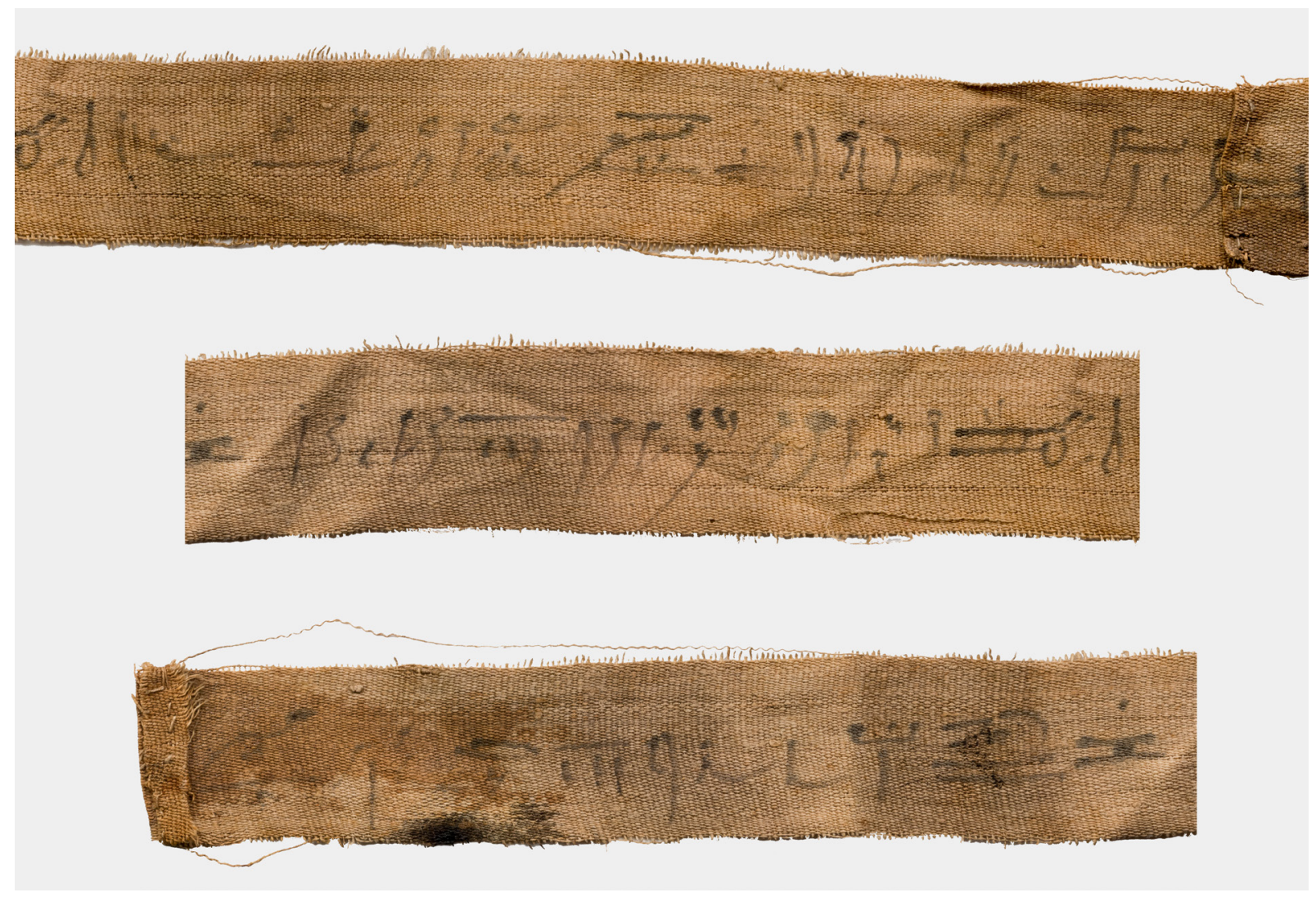

Taf. 3 Spruch 173 (Pleyte) 